\title{
Advances in miRNA-Mediated Mucin Regulation
}

\author{
Shiv Ram Krishn • Surinder K. Batra • \\ Sukhwinder Kaur
}

Published online: 15 January 2015

(C) Springer International Publishing AG 2015

\begin{abstract}
MicroRNAs (miRNAs) are an important class of small non-coding RNAs that direct post-transcriptional gene regulation by different mechanisms. Mounting evidences from numerous studies in the last decade have unraveled that deregulated miRNAs, and their cognate target expressions are strongly implicated in the carcinogenesis. Recent advances have highlighted miRNA-mediated regulation of mucins that have critical role in inflammation and cancer biology. The aberrant expression and differential glycosylation of mucins cause tumorigenesis, metastasis, chemoresistance and poor outcome of cancer patients, thus recognizing them as attractive therapeutic targets. Though current mucin-based therapies (antibodies, vaccines, immunotherapy, peptide inhibitors) have shown preclinical utility but only a handful promise for clinical transferability. In this context, understanding miRNA-mediated modulation of multiple mucin(s) expression and function gives a new hope for future antitumor therapeutics. Herein, we reviewed miRNA biogenesis, mechanism of action, and their role in tumor development. Further, we provide an overview of miRNA-mediated mucin regulation and their application as anticancer therapeutics.
\end{abstract}

Keywords miRNA $\cdot$ Mucins $\cdot$ Mucin regulation $\cdot$ Cancer therapy

$\begin{array}{ll}\text { Abbreviations } \\ \text { 2'-MOE }^{\prime} \text { 2'-Methoxyethyl } \\ \text { AMOs } & \text { Anti-miRNA oligonucleotides } \\ \text { ASOs } & \text { Antisense oligonucleotides } \\ \text { CDX2 } & \text { Caudal-related homeobox protein } \\ \text { CRC } & \text { Colorectal cancer } \\ \text { ER } \beta 1 & \text { Estrogen receptor } \beta 1 \\ \text { HMOX1 } & \text { Hemeoxygenase-1 } \\ \text { IL-13 } & \text { Interleukin-3 } \\ \text { IL-8 } & \text { Interleukin-8 } \\ \text { LNA } & \text { Locked nucleic acid } \\ \text { MAPK } & \text { Mitogen-activated protein kinase } \\ \text { miRNA } & \text { microRNA } \\ \text { NLE } & \text { Neutral lipid emulsions } \\ \text { PAMAM } & \text { Polyamidoamine } \\ \text { PC } & \text { Pancreatic cancer } \\ \text { PLGA } & \text { Polyurethanes poly (lactic-co-glycolic acid) } \\ \text { PNA } & \text { Peptide nucleic Acid } \\ \text { SNALPs } & \text { Stable nucleic acid lipid particles } \\ \text { TsmiR } & \text { Tumor suppressor microRNA } \\ \text { UCB } & \text { Urothelial carcinoma of bladder }\end{array}$

\section{Introduction}

Prevention and Therapeutic Agents

S. R. Krishn · S. K. Batra $(\bowtie) \cdot$ S. Kaur $(\bowtie)$

Department of Biochemistry and Molecular Biology, University of

Nebraska Medical Center, Omaha, NE 68198, USA

e-mail: sbatra@unmc.edu

e-mail: skaur@unmc.edu

S. K. Batra

Fred and Pamela Buffett Cancer Center, Eppley Institute for Research in Cancer and Allied Diseases, University of Nebraska Medical Center, Omaha, NE 68198-5870, USA
Cancer is one of the leading causes of death worldwide with 1 , 658,370 new cancer cases and 589,430 cancer deaths expected in the United States alone in 2015 [1]. Over the years, laudable progresses have been made in understanding of cancer initiation, progression, and metastasis; but till date, we are far behind in prolonging the median survival of the cancer patients. The quest for newer strategies to target tumors led to the emergence of miRNAs as next generation potential 
cancer therapeutics. Discovered in 1993 by Ambros and colleagues, these small, evolutionarily conserved endogenous non-coding RNAs (21-23 nucleotides) regulate gene expression typically by binding to the 3 '-untranslated region (UTR) of mRNAs and causing inhibition of translation and/or mRNA degradation $[2,3]$. The miRNAs by impacting over $30 \%$ of all protein-coding genes play important role in numerous cellular processes including embryonic development, cell differentiation, metabolism, proliferation, apoptosis, and stress response in diverse invertebrate and vertebrate organisms including humans [4]. Additionally, aberrant expression of miRNAs has been associated with numerous pathological conditions including cardiovascular diseases, neurological disorders, diabetes, and viral infections [5]. Altered expression of miRNAs has been critically implicated in the pathogenesis of cancers [4]. Moreover, the expression patterns of miRNAs are unique to each tumor type and to their tissue of origin [6]. It is now well established that miRNAs impact the development of cancer by altering the expression of both oncogenes and tumor suppressor genes [6]. Interestingly, recent studies have unraveled unprecedented role of miRNAs in the regulation of various mucins.

Mucins are high molecular weight multifunctional glycoproteins designed as external environment sensors to act for cellular protection. Based on their structure, mucins are classified into transmembrane/membrane-bound (MUC1, MUC3A, MUC3B, MUC4, MUC12, MUC16, and MUC17) having integral transmembrane domain and secreted/gelforming mucins (MUC2, MUC5AC, MUC5B, MUC6, and MUC19) [7]. The bulky extracellular part of membrane tethered mucins is comprised of several unique domains that modulate various biological properties by selective interactions with various ligands, cell-surface proteins, and the components of extracellular matrix [8]. Their relatively short cytoplasmic tails associate with cytoskeletal elements, cytosolic adaptor proteins, and/or participate in signal transduction [9]. Secreted mucins, on the other hand, lack hydrophobic transmembrane domain and form a mucus layer on the apical surfaces of healthy epithelial cells to protect them from external environmental stresses $[10 \bullet \bullet$.

Various epithelial malignancies (breast, lung, pancreatic, ovarian, and bladder) are characterized by deregulated expression of mucins expression and glycosylation. Altered mucins by virtue of physical interactions or by regulating cellular signaling cascades promote malignant transformation, cancer cell growth, cell invasiveness, metastasis, drug resistance, and decreased immune surveillance [11]. The cancer cell specific overexpression, multiplicity of epitopes, and posttranslational modifications of mucins make them worthy candidates for targeted therapeutics [12]. Currently, mucin-based therapies in clinical and preclinical studies rely on: (i) mucin silencing by RNA interference, (ii) mucin promoter driven suicide gene therapy, (iii) antibody-targeted therapies, (iv) recombinant peptide vaccines to stimulate the cell mediated immunity dependent tumor cell killing, (v) small-size inhibitory peptides that block mucin cytoplasmic tail interaction with other signaling molecules, (vi) and mucin mimetic to inhibit cytoplasmic tail oligomerization, thus preventing its translocation to the mitochondria or nucleus $[10 \bullet \cdot]$. Despite mucins being the potent targets for therapy, lack of mucinspecific small molecule inhibitors, possible diluted efficacy of the targeting agent (mucin-specific antibodies, peptides, or aptamers) due to large pools of circulating N-terminal ectodomains and tumor heterogeneity with regard to mucin expression, demands discovery of newer means to specifically target cancer specific mucins. Considering emerging implications of miRNA in therapies, in the present article, we tend to highlight miRNA-mediated regulations of mucins and their potential utility as a therapeutic modality.

In this review, we briefly describe emerging roles of miRNAs in cancers biology, their biogenesis, recent advances in miRNA-based therapeutic approaches and miRNAmediated mucin regulation (MUC1, MUC4, MUC16, MUC17, MUC2, and MUC5AC) (Table 1) in different malignancies. Overall, we aim to understand the potential application of miRNA centered therapeutics to modulate mucin expression.

\section{MiRNAs in Cancer}

Over the last two decades, many miRNAs have been implicated in various human malignancies. Observations from genome wide studies revealed a differential miRNA profile in malignant cells in comparison to normal cells. More and more miRNAs are being located at genomic regions involved in cancers, chromosomal break points, as well as in the minimal areas of the loss of heterozygosity/amplifications [31]. The initial findings that two microRNAs, miR-15 and 16, were downregulated or deleted in most patients with chronic lymphocytic leukemia-launched miRNAs to the center stage of molecular oncology [32]. From a study done in 334 patient samples of various cancer types, it was observed that developmental lineage and differentiation state of the tumors can be distinguished using miRNA expression profile of these samples [33]. Later on, several groups reported an altered expression of miRNA in various cancers. Based on their functional attributes in cancer, the miRNA have been categorized into tumor suppressor miRNAs (TsmiRs), oncogenic miRNA (OncomiRs), MetastamiRs, and AngiomiRs. TsmiRs are downregulated in cancers due to different genetic mechanisms as epigenetic modification, somatic mutation, biallelic deletion, loss of heterozygosity, and altered processing and are known to promote tumor formation. Numerous TsmiRs have been observed to inhibit tumor growth on ectopic expression; examples include miR-26, miR-29, miR-150, miR-200, miR- 
Table 1 Functional implications of mucin regulation by miRNAs

\begin{tabular}{|c|c|c|c|}
\hline Mucin & miRNA & Associated putative role & Reference \\
\hline \multirow[t]{8}{*}{ MUC1 } & \multirow[t]{4}{*}{ miR-145 } & - TsmiR: downregulated in breast cancer & \multirow[t]{2}{*}{ [13] } \\
\hline & & - Downregulates MUC1 and inhibits invasion and metastasis of breast cancer cells & \\
\hline & & - TsmiR: downregulated in ovarian cancer & \multirow[t]{2}{*}[14]{} \\
\hline & & - Impacts ovarian cancer cell growth and metastasis by decreasing MUC1 levels & \\
\hline & $\operatorname{miR}-1226$ & - TsmiR: downregulates MUC1 expression in breast cancer cells to induce apoptosis & [15] \\
\hline & \multirow[t]{2}{*}{$\operatorname{miR}-125 b$} & $\begin{array}{l}\text { - TsmiR: downregulated in breast cancer } \\
\text { - Reduces MUC1 levels resulting in decreased growth and increased apoptosis }\end{array}$ & [16] \\
\hline & & - Suppresses translation of MUC1 in prostate cancer cells & [17] \\
\hline & $\operatorname{miR}-455-3 p$ & $\begin{array}{l}\text { - Decreased expression associated with increased MUC1 expression in differentiated } \\
\text { bronchial epithelial cells }\end{array}$ & [18] \\
\hline MUC2 & miR-9 & $\begin{array}{l}\text { - OncomiR: repressing expression of CDX2 and its downstream target MUC2 resulting } \\
\text { in increased cell proliferation in gastric cancer cells }\end{array}$ & [19] \\
\hline \multirow[t]{5}{*}{ MUC4 } & Let-7i and Let- $7 b$ & $\begin{array}{l}\text { - The ectopic expression of let-7i and let-7b lead to downregulation of MUC4 expression } \\
\text { in gemcitabine resistant urothelial carcinoma cells }\end{array}$ & {$[20]$} \\
\hline & $\operatorname{miR}-150$ & $\begin{array}{l}\text { - TsmiR: downregulated in pancreatic cancer } \\
\text { - Decrease pancreatic cancer cell growth, invasion, and metastasis by decreasing MUC4 } \\
\text { expression and associated signaling }\end{array}$ & {$[21,22 \bullet \bullet]$} \\
\hline & miR-150-5p & $\begin{array}{l}\text { - TsmiR: low expression in colorectal cancer } \\
\text { - Ameliorates colorectal cancer cell migration and invasion by reducing MUC4 } \\
\text { expression at both mRNA and protein levels }\end{array}$ & [23] \\
\hline & miR-200c & $\begin{array}{l}\text { - TsmiR: downregulated in pancreatic cancer } \\
\text { - Inhibits pancreatic cancer cell growth and metastasis by a reducing MUC4 } \\
\text { mRNA and protein levels }\end{array}$ & {$[24 \bullet \bullet]$} \\
\hline & $\operatorname{miR}-219-1-3 p$ & $\begin{array}{l}\text { - TsmiR: downregulated in pancreatic cancer } \\
\text { - Suppress MUC4 and its downstream signaling to inhibit pancreatic cancer cell } \\
\text { proliferation and migration }\end{array}$ & [25] \\
\hline \multirow[t]{2}{*}{ MUC5AC } & miR-146a & - Decreases neutrophil elastase stimulated MUC5AC overproduction in lung epithelial cells & [26] \\
\hline & $\operatorname{miR}-378$ & $\begin{array}{l}\text { - OncomiR: its increased expression increases MUC5AC, VEGF, and IL-8 expression leading to } \\
\text { increased proliferation, migration of lung cancer cells }\end{array}$ & {$[27]$} \\
\hline \multirow[t]{4}{*}{ MUC16 } & miR-92 & - TsmiR: decrease in its expression restores MUC16 levels in breast cancer cells & {$[28]$} \\
\hline & miR-100 & $\begin{array}{l}\text { - Low levels in epithelial ovarian cancer tissues correlated with higher serum } \\
\text { CA125 (MUC16) expression level }\end{array}$ & [29] \\
\hline & miR-145 & $\begin{array}{l}\text { - Inverse relationship to MUC16 (CA125) levels observed } \\
\text { in serum samples from ovarian cancer patients }\end{array}$ & {$[14]$} \\
\hline & miR-200c & $\begin{array}{l}\text { - Decreased expression in pancreatic cancer cells resulted in reduced expression of MUC16 } \\
\text { mRNA and protein by directly targeting its mRNA coding sequence }\end{array}$ & {$[24 \bullet \bullet]$} \\
\hline MUC17 & $\begin{array}{l}\text { miR-17, miR-20a, miR-20b, } \\
\text { miR-30c, miR-30e }\end{array}$ & $\begin{array}{l}\text { - Significant correlation with MUC17 expression in colon and pancreatic cancer cell } \\
\text { lines. Putative role needs further validation }\end{array}$ & {$[30]$} \\
\hline
\end{tabular}

145, miR-1226, miR-100, miR-92, and the let7 family members [34]. OncomiRs: OncomiRs represent a class of miRNAs whose constitutive overexpression results in accelerated tumor formation and progression. The first identified oncomiR was polycistronic miR-17-92 cluster that is located in the chromosomal locus 13q31-q32 known to be frequently amplified in solid and hematopoietic tumors [35]. The evidences for overexpression of miR-17-92 have also been documented in several solid malignancies of the breast, lung, colon, stomach, pancreas, and prostate [36]. Overexpression of another oncomiR miR-21 is associated with advanced clinical stage, tumor growth, lymphnode metastasis, and poor prognosis in breast cancer samples [37]. MetastamiRs: The miRNAs involved in the acquisition of invasive abilities by controlling loss of cellular adhesion regulate genes associated with epithelial to mesenchymal transition (EMT), entry into and exit from the vascular system, survival in the vascular system and at distant sites are termed as metastamiRs. Epigenetic silencing of miR-200 results in ZEB-1 and ZEB-2 upregulation mediated repression of E-cadherin [34]. In addition to this, miR-9 is known to directly inhibit E-cadherin, miR155 and miR-31 regulate RhoA and miR-181, which inhibits the tumor suppressor metalloprotease inhibitor 3 (TIMP3) [34]. AngiomiRs: Tumor cells promote proliferation and migration of endothelial cells resulting in increased angiogenesis. In different cancers, miR-15a/16-1 cluster, miR-145, and miR-143 are known to inhibit angiogenesis [2, 38]. miRNA and cancer stem cells: Accumulating evidences from recent 
studies have shown involvement of miRNAs as let-7, miR-30, miR-296, miR-134, miR-470, and miR-34 family in regulation of genes associated with pluripotency and stem cell function as OCT4, NANOG, SOX2, NOTCH, and BCL2 as reviewed in [2].

\section{MiRNA Biogenesis and Mechanisms of Action}

The multistep biosynthesis of miRNAs involves both the nuclear and cytoplasmic components [39]. The primary miRNA precursor, pri-miRNA (several kilobases long), is first transcribed by RNA polymerase II as double-stranded stemloop structure (5' 7-methyl guanylate $(\mathrm{m} 7 \mathrm{G})$ capped and 3'polyadenylated) [40]. These pri-miRNAs are then processed in the nucleus by Drosha/DGCR8 (a ribonuclease III (RNase III) endonuclease) into 65-70 bp stem-loop precursor miRNA (pre-miRNA) [41]. The pre-miRNA is then transported from the nucleus to the cytoplasm by Exportin-5-RanGTPdependent mechanism and is further processed by RNase III endonuclease, Dicer/TRBP into a mature 17-25 bp RNA duplex [42]. The miRNA duplexes enter the miRNAinduced silencing complex (miRISC) where the helicase activity of Argonaute (Ago) protein causes the RNA duplex to unwind [43]. The mature, single-stranded miRNA (one of the strand) is retained into the RNA-induced silencing complex (RISC) and directs the complex to target $3^{\prime}$ and/or 5'-UTR of mRNA [44]. Partial complementarity between the seed region of miRNA and the 3'-UTR region of the target gene leads to deadenylation and destabilization of mRNA, whereas perfect complementarity results in target mRNA degradation [45]. This promiscuity of complementarity is a unique feature of miRNA that allows one miRNA strand to recognize an array of mRNA. Through a combination of different mechanisms, miRNAs have a significant effect on protein synthesis in cells. MiRNAs repress initiation of translation directly, or sequester mRNA in regions of the cell where low protein production takes place (e.g., P-bodies), may cause ribosome stalling, drop off ribosome from mRNA leading to protein truncation or recruit proteases that degrade protein as translation is underway [34].

\section{Regulation of Mucin by miRNAs}

Normal tissue homeostasis requires tight regulation of mucin expression. However, several environmental and/or intrinsic insults compromise this regulation leading to aberrant expression of mucins in diverse inflammatory and pathological disorders including cancer. Deregulated expression of transmembrane mucins (MUC1, MUC4, MUC16, and MUC17) and of secretory mucins (MUC2 and MUC5AC) is a prominent characteristic that has been well studied in various types of cancers and inflammatory diseases. They impact various aspects of tumor including growth, metastasis, and oncogenic signaling, thus acting as prominent candidates for therapeutic targeting. In recent times, miRNAs have emerged as important regulator for altered mucin expression during malignant development. MiRNA-mediated modulation of key mucins (MUC1, MUC4, MUC16, MUC17, MUC2, and MUC5AC) involved in oncogenic development and progression of cancer along with emerging technologies used for their targeting is described below.

\section{MUC1}

Normal ductal epithelial cells of breast, lung, gastrointestinal tract, and pancreas basally express transmembrane glycoprotein MUC1. However, aberrantly glycosylated MUC1 is overexpressed in a variety of epithelial malignancies (ovarian, breast, lung, and pancreatic) and is a poor prognostic factor. Overexpressed MUC1 plays multifaceted roles by impacting inflammatory milieu of tumor, cancer cell proliferation, resistance to apoptosis, invasion, angiogenesis, and chemoresistance [46], thereby making it an attractive target for therapies.

MUC1-altered expression in different malignancies have uncovered its regulation by multiple miRNAs (miR-145, miR-1226, miR-125b, miR-551b, and miR-149 (Table 1). The miR-145 is downregulated in breast cancer [47] and colon cancer [48] and is hypothesized to be a putative tumor suppressor. Recently, Sachdeva et al. highlighted that miR-145 downregulates invasion and metastasis of metastatic breast cancer cells by directly targeting 3 '-UTR of MUC1 to suppress its expression resulting in reduced expression of $\beta$ catenin and cadherin-11 [13]. Further, Wu et al. demonstrated that miR-145 is downregulated in ovarian cancer tissues, cell lines, and serum samples in comparison to normal controls. They showed that miR-145 functions as a tumor suppressor in ovarian cancer by modulating in vitro and in vivo ovarian cancer growth and invasion by negatively regulating p70S6K1 and MUC1 protein levels by directly targeting their 3'-UTRs [14].

The miR-1226 downregulates the expression of MUC1 oncoprotein in breast cancer cells, induces cells for apoptosis by increasing ROS production, and loss of mitochondrial transmembrane potential [15]. Further, different findings collectively indicate that miR-125b is aberrantly regulated in human cancers where it contributes to oncogenesis (pancreatic cancer [49] and acute myeloid leukemia [50]) or functions as a tumor suppressor (breast cancer [51]) in a cell context manner. Notably, Rajabi et al. reported that miR-125b is downregulated in breast cancer cells (BT-549 and ZR-75-1) and functions as a tumor suppressor by reducing the expression of transmembrane oncoprotein MUC1 leading to reduced cell growth and induction of apoptosis [16]. Besides, the same group demonstrated that miR-125b suppresses translation of oncoprotein MUC1 (overexpressed in aggressive human 
prostate cancers) in both androgen dependent (LNCaP) and independent (DU145) prostate cancer cells [17]. MUC1 plays a very important role in protecting normal lung epithelial cells from pathogens. Interestingly, Martinez-Anton et al. reported that decreased expression of miR-455-3p in differentiated pseudostratified epithelium of human bronchial epithelial cells results in 136-fold increase in expression of epithelial cell marker MUC1 by targeting $3^{\prime}$-UTR in comparison to undifferentiated monolayer signifying its importance for differentiation [18].

In another study, $\mathrm{Xu}$ et al. revealed that increased miR-551b expression in lung cancer cells with acquired chemoresistance inhibited the expression of catalase and increased ROS accumulation and MUC1 expression. Further, MUC1 mediates chemoresistance via activation of cell survival (EGFR/Akt/c-FLIP/COX2) cascade. [52].

Taken together, these studies highlight that by increasing the expression of tumor suppressor miRNAs or by decreasing the expression of oncogenic miRNAs using different miRNAbased approaches, MUC1 expression can be modulated to decrease tumor burden. At present, it is difficult to predict among various miRNA, the most effective miRNA in any given malignancy, but by targeting multiple miRNAs at a time as in case of breast cancer (miR-145, miR-125b, miR-1226), MUC1-mediated oncogenic effects can easily be impacted.

\section{MUC4}

MUC4 is a large transmembrane mucin that is often overexpressed in diverse epithelial tumors (breast, ovarian, and pancreatic) [53]. MUC4 acts as a ligand for receptor tyrosine kinase-Her2, thereby mediating activation of downstream mitogen-activated protein kinase (MAPK), phosphoinositide-3-kinase (PI3K)/Akt and c-Src/FAK family kinase pathways to regulate cell proliferation and metastasis [53]. MUC4 mediated phosphorylation of BAD results in sequestration of $14-3-3 \zeta$ in the cytoplasm leading to its anti-apoptotic effects and gemcitabine resistance [54]. MUC4 with its oncogenic impact on cell proliferation, invasion, and drug resistance is a potential therapeutic target.

Relevant studies associated with regulation of MUC4 mucin in different cancers have previously been reported, but recent evidences about MUC4 regulation by miRNAs holds immense therapeutic potential. In this regard, Wang et al. highlighted the metastasis inhibiting role of miR-150-5p by its direct targeting of transmembrane mucin MUC4. Low expression of miR-150-5p has been associated with a worse response to adjuvant chemotherapy and shorter survival in colorectal cancer (CRC) patients [55]. The tumor suppressive utility of miR-150-5p mimic as a novel agent for treatment of colorectal cancer was displayed by its potential to ameliorate
CRC cell migration and invasion mediated by suppression of transmembrane mucin MUC4 expression at both mRNA and protein levels through its direct binding to MUC4 3 '-UTR [23]. Further, findings from Srivastava et al. established miR-150 as a novel regulator of MUC4 and a tumor suppressor miRNA in pancreatic cancer (PC). Their study revealed that downregulation of miR-150 in PC tissues has an inverse relationship to MUC4 endogenous protein expression. They demonstrated that overexpression of miR-150 may have therapeutic effects in PC as it results in decreased expression of MUC4, downregulation of MUC4-associated signaling, and concomitant inhibition of PC cell growth, clonogenicity, migration, invasion, and enhanced intercellular adhesion [21]. Interestingly, Lahdaoui et al. observed that miR-219-1-3p is downregulated in pancreatic cancer-derived cell lines, PC precursor lesion in KrasG12D-driven PC mouse model, and PC tissues. MiR-219-1-3p mediated suppression of MUC4 and its downstream signaling, inhibit pancreatic cancer cell proliferation in vitro and in vivo and migration in vitro [25]. Furthermore, study by Radhakrishnan et al. demonstrated that miR-200c may inhibit PC cell growth and metastasis by a significant downregulation of MUC4 mRNA and protein expression through direct targeting the mRNA-coding sequences of MUC4 [24••].

Additionally, Kozim et al. drew attention to the potential therapeutic strategy for improving the efficacy of gemcitabine in urothelial carcinoma of the bladder (UCB) by emphasizing a role for miRNAs-1290 and 138 (increased expression), miRlet- $7 \mathrm{i}$, and let-7b (decreased expression) in gemcitabine resistant cells through the modulation of oncogenic mucin MUC4. The ectopic expression of let-7i and let-7b lead to downregulation of MUC4 expression in gemcitabine resistant cells [20].

\section{MUC16}

MUC16 is a transmembrane mucin overexpressed in ovarian, uterine, pancreatic, breast, and biliary cancers. It is associated with tumor progression, metastasis, and poor prognosis [56]. Mechanistically, CA125/MUC16 interact with E-cadherin/- $\beta$ catenin complex to modulate epidermal growth factor receptor (EGFR) activation, cellular localization and its downstream targets Akt and ERK1/2 signaling and in-turn promotes cell dissemination and cell motility [57]. Additionally, cytoplasmic domain of MUC16 through its interaction with ezrin/ radixin/moesin proteins causes cytoskeleton reorganization [56]. Our group has recently reported that MUC16 activates STAT3 and c-Jun signaling pathway to promote cell proliferation of breast cancer cells by physically interacting with ERM domain containing Jak2 protein [58]. Though structure and function of MUC16 have been studied to some extent but regulation of its expression is still unexplored. Knowledge 
gained by understanding its regulation can be utilized for therapeutic targeting in different malignancies.

Radhakrishnan et al. unraveled that transfection of pancreatic cancer cells S2.028 and T3M-4 with miR-200c resulted in reduced expression of MUC16 mRNA and protein by directly targeting its mRNA coding sequence [24••]. Further, AlNakhle et al. observed that manipulation of miR-92 expression in vitro modulates ER $\beta 1$ and MUC16 expression in MCF7 breast cancer cells. They demonstrated that miR-92 silencing significantly restored MUC16 expression in breast cancer cells [28]. Recently, Peng et al., observed significantly low levels of miR-100 in epithelial ovarian cancer tissues (EOC) compared to adjacent normal tissues. Further, its level closely correlated with higher serum CA125 (MUC16) expression level, lymph node involvement, and shorter overall survival of EOC patients [29]. Similarly, the converse relationship between miR-145 and MUC16 (CA125) levels was observed in serum samples from ovarian cancer patients [14]. This necessitates a further investigation about MUC16 regulation by miR-145, as the information gained can be utilized for miR-145-mediated dual targeting of overexpressed membrane-bound mucins MUC1 and MUC16 in ovarian cancer.

\section{MUC17}

Membrane-bound mucin MUC17 (normally expressed in digestive tract including duodenum, ileum, and transverse colon) is lost during inflammatory conditions of colon like colitis and Crohn's disease, underscoring its potential protective role in maintaining colon homeostasis [59]. Besides, it is aberrantly expressed in PC compared to no expression in normal pancreas, pancreatitis and identified as an independent prognostic factor associated with lymphnode metastasis of PCs $[60,61]$. As functional role of MUC17 in cancer pathology is still obscure therefore, understanding the mechanisms underlying its aberrant expression can help to devise new diagnostic and therapeutic modalities. In one such revelation using miRNA microarray analysis in 11 cancer cell lines, Kitamoto et al. reported five candidate microRNAs (miR-17, miR-20a, miR-20b, miR-30c, and miR-30e) have significant relationship to potential post transcriptional regulation of MUC17 [30]. The therapeutic potential of these miRNAs for MUC17 modulation in different cellular contexts needs further in vitro and in vivo evaluation.

\section{MUC2}

MUC2 is a gel-forming secretory mucin that is normally expressed in organs including colon, small intestine, and respiratory tract. Deregulated expression of MUC2 has been associated with CRC development and development of some type of lung cancer $[62,63]$. Most notably, the tumor suppressor role of Muc2 were observed in MUC2 knockout (Muc2 $^{-1}$ ) mice that frequently developed adenomas in the small intestine and progress to invasive adenocarcinoma and rectal tumors [64]. Additionally, MUC2 expression decrease progressively with increasing stages of gastric cancer [65]. Interestingly, Rotkrua et al. in their studies revealed that miR9 might be responsible for gastric carcinogenesis by repression of CDX2 (caudal-related homeobox protein) and its downstream target like secretory mucin MUC2 resulting in promotion of cell proliferation in gastric cancer cells (MKN45 and NUGC-3) [19]. Thus, gastric carcinogenesis can be slowed down by indirect targeting of mucin MUC2 through identification of CDX2-preserving anti-miR-9 as a therapeutic agent.

\section{MUC5AC}

MUC5AC is a secretory mucin that is typically expressed by gastric mucosa and airway pathways. Hypersecretion of secretory mucin MUC5AC is an important manifestation observed in patients with chronic inflammatory airway diseases [66]. Deregulated expression of MUC5AC has been reported in many cancers including PC, colorectal cancer, lung adenocarcinoma, and cholangiocarcinoma [53]. Its overexpression has been associated with early post-operative metastasis in non-small cell lung cancer patients [67] and worse prognosis for lung adenocarcinoma, cholangiocarcinoma [68], and PC patients [69]. Mechanistically, MUC5AC has been shown to affect E-cadherin-mediated invasion and metastasis in PC and CRC cells [53]. A plethora of studies has been done to understand the mechanisms behind regulation of MUC5AC in different pathologies. However, limited observations are available regarding miRNA-mediated regulation of MUC5AC. Zhong et al. explored the therapeutic potentials of manipulating miR-146a for the management of mucus overproduction during inflammatory airway diseases. MiR146a downregulated MUC5AC production from airway epithelial cells that were stimulated by neutrophil elastase through JNK and NF-KB signaling [26]. Moreover, Interleukin-13 (IL-13) plays a critical role in airway inflammation-associated MUC5AC overproduction while the mechanisms underlying this process are not entirely elucidated [70]. Kumar et al. observed that intranasal delivery of Let-7 mimic (oligonucleotides designed by chemically modifying $3^{\prime}$ terminal in both strands with 2'-O-methoxy substitution) in a mouse model of allergic airway inflammation alleviates asthma features like reduction in IL-13 levels, airway hyperresponsiveness, and airway inflammation. Interestingly, Let-7 mimic also reduced goblet cell metaplasia (airway mucin content) determined by periodic acid Schiff staining [71]. Interestingly, Skrzypek et al. displayed that overexpression of hemeoxygenase-1 (HMOX1) not only decreased the expression of miR-378 (Oncomir/Angiomir) but 
also reversed miR-378 mediated increased expression of MUC5AC, vascular endothelial growth factor (VEGF), interleukin-8 (IL-8), and Ang-1. This resulted in decreased proliferation, migration, and angiogenic potential of NCIH292 lung cancer cells both in vitro and in vivo. Thus, this study highlights a prospective therapeutic strategy to inhibit miR-378-mediated impact on lung cancer cell growth and metastasis [27].

\section{MiRNA and Cancer Therapy}

Therapeutic modulation of miRNAs is broadly executed at two different levels: by reducing/increasing their levels to affect their downstream targets and/or to interfere with the miRNA/ mRNA interaction to obtain higher degrees of specificity [45]. The two most prominent challenges associated with the manipulation of miRNA function are (a) identification of molecules to inhibit or "mimic" mature miRNAs and (b) efficient delivery of these molecules to specific targeted sites. Inhibition of miRNAs overexpressed in cancer can be achieved by base pairing them with complementary oligonucleotide sequences termed as antisense oligonucleotides (ASOs) [5]. Using this approach, multiple steps in the biogenesis of miRNA can be targeted including targeting the loop structure of the pre-miRNA [72] or inhibition of processing of pri-miRNAs and pre-miRNAs by Drosha and Dicer, respectively by morpholino ASOs [73]. They also act as target protectors by binding and protecting the target sequence on the mRNA by denying access to miRNA. More recent approach is to target mature miRNAs directly using anti-miRNA oligonucleotides (AMOs). These AMOs act by near irreversibly binding to mature miRNA and prevent them to recognize its cognate mRNA sequence. Further, chemical modification of 2'-hydroxyl of the ribose in these AMOs to 2'-O-methyl along with modification of the phosphodiester backbone to phosphorothioate not only enhanced binding affinity to miRNA but also protected from nuclease degradation [74]. The activity of AMOs against target miRNA can be further enhanced by addition of methoxyethyl (2'-MOE) or fluorine (2'-F) groups when compared to simpler 2'-O-methyl modification. Moreover, the strongest affinity to miRNAs can be obtained with Locked nucleic acids (LNAs) modified AMOs whose ribose moiety has an extra bridge connecting the $2^{\prime}$ oxygen and 4'-carbon resulting in a locked conformation yielding more thermodynamic stability and target specificity to ribonucleotides both in vitro and vivo [74]. Peptide nucleic acids (PNAs) having synthetic polymers similar to RNA and DNA are becoming excellent candidates for antisense therapies.

In addition to normal AMOs that have only one binding site for target miRNA, another class of miRNA inhibitors developed is miRNA sponges. These competitive inhibitors consist of a strong promoter driven expression of an artificial transcript that provide target sites to attract multiple miRNA family members. It thus inhibits the activity of miRNAs by letting endogenous targets escape the inhibition [75]. Further, re-expression of miRNAs downregulated in cancer involves strategies harnessing miRNA mimics and viral expression constructs encoded miRNAs. The miRNA mimic is doublestranded RNA molecules that can be chemically modified (addition of methyl, methoxyethyl, fluorine, 3 '-benzene-pyridine) to protect them from nucleases, decrease innate immune system activation and off target effects [74]. Alternatively, expression of pri-miRNA mimic using viral expression construct provides a more stable miRNA replacement.

Nevertheless, widespread in vivo application of miRNA inhibitors and mimics is hindered by their lack of tissue specificity. Negative charge of these ASOs, AMOs, and mimics interfere in their crossing the cell membrane readily. Therefore, to improve the delivery of this potential therapeutics in cellular and animal models several technical approaches have been developed. Different viral vectors (adenovirus, retrovirus, lentiviruses) due to their high transduction efficiency have been extensively used for delivering miRNAs to cancer cells. In addition to this, the cellular intake of ASOs, AMOs/miRNA mimics, and their protection from the hostile microenvironment can also be improved by encasing them in cationic liposomal nanoparticles [76], combining with neutral lipid emulsions (NLE) [77] and by using stable nucleic acid lipid particles (SNALPs) [78]. Moreover, delivery to tumor cells can also be accomplished by using polymer (Polyurethanes, poly (lacticco-glycolic acid) PLGA and polyamidoamine (PAMAM) dendrimers)-based nanoparticles. Delivery of miRNAs in such lipid-based vehicles may have less toxicity and immune response. Using this technique, Arora et al. investigated whether restoration of miR-150 could serve as a useful strategy for PC therapy. For this, they utilized polyethyleneimine (a cationic polymer) and mixed it with poly (D, $L$-lactide-co-glycolide) (PLGA) to develop a nanoformulation of miR-150 (miR-150$\mathrm{NF}$ ) and evaluated its therapeutic efficacy in vitro. The efficient intracellular delivery of miR-150 mimics by miR-150-NF resulted in a significant downregulation of MUC4 expression concomitant with inhibition of HER2 and downstream signaling implicating in suppressed growth, clonogenicity, motility, and invasion of PC cells. Together, these results highlighted a safe and effective nanovector platform for miR-150 delivery to pancreatic tumor cells $[22 \bullet \bullet]$.

Besides, targeted delivery to specific tissues can be done by linking tumor specific ligands to nanoparticles that are directed to tumor cells via active (conjugating with compounds that have a specific affinity to tumors) or passive (using size of nanoparticles and tumor vasculature) targeting. Dai et al. utilized MUC1 as a tumor specific ligand to target ovarian cancer cells and devised a miRNA-based chimera composed of MUC1 aptamer and miR-29 (Chi-29b) to inhibit DNA methyltransferases expression [79]. They successfully demonstrated that Chi-29b chimera exhibits its antitumor and antichemoresistant roles through downregulation of MAPK4 
and IGF1 expressions and activation of PTEN signaling in OVCAR-3 tumors [80]. Further, chimera of MUC1 aptamer and let-7i miRNA was specifically delivered to OVCAR-3 cells. It resulted in reversal of the paclitaxel resistance and induced cell apoptosis, inhibit cell proliferation by reducing expression of Cyclin D1, Cyclin D2, Dicer 1, and PGRMC1 [81].

\section{Conclusion(s) and Perspectives}

Mucins are important biomolecules for cellular homoeostasis and protection of epithelial surfaces. Their aberrant expressions in various malignancies make them attractive target(s) for the development of antibodies, vaccines, radioimmunoconjugates, and therapeutic inhibitors. The anti-mucin therapeutic modalities hold immense possibility and promise, but their transition from bench to bedside necessitates identifying novel ways to target them. Though miRNA-mediated mucin regulation has just started to emerge, both miRNA mimics and anti-miRs give us hope to control differential mucin expression in various malignancies. Importantly, elucidation of the functional outcome concomitant with miRNA-mediated modulation of mucin expression will help in devising newer therapeutic approaches to counter heterogeneity of cancers in terms of mucin expression. In this regard, advancements have been made not only utilizing mucins as ligands for specific targeting of miRNAs but also employing nanovector based platforms for safe and effective delivery of miRNAs to target cancer specific mucin expression. MiRNA targeted therapy have been utilized to interfere with the mucin function. Future clinical trials should provide new insights into the safety and efficacy of the currently developed miRNA-based mucin modulating anticancer therapies. In the long term, more studies need to be focused on identifying miRNAs targeting multiple mucins in a tumor specific manner along with the development of safe and specific methods to deliver such miRNAs for improved cancer treatment and management.

\section{Compliance with Ethics Guidelines}

Conflict of Interest Shiv Ram Krishn, Surinder K. Batra, and Sukhwinder Kaur declare that they were, in parts, supported in part by the grants from National Institutes of Health (RO1 CA183459, EDRN UO1 CA111294, SPORE P50 CA127297, and TMEN U54 CA163120).

Human and Animal Rights and Informed Consent This article does not contain any studies with human or animal subjects performed by any of the authors.

\section{References}

Papers of particular interest, published recently, have been highlighted as:

• Of major importance

1. Siegel R, Miller K, Jemal A. Cancer statistics, 2015. Cancer J Clin. 2015. doi:10.3322/caac. 21254

2. Kong YW, Ferland-McCollough D, Jackson TJ, Bushell M. microRNAs in cancer management. Lancet Oncol. 2012;13(6): e249-58.

3. Lee RC, Feinbaum RL, Ambros V. The C. elegans heterochronic gene lin-4 encodes small RNAs with antisense complementarity to lin-14. Cell. 1993;75(5):843-54.

4. Croce CM. Causes and consequences of microRNA dysregulation in cancer. Nat Rev Genet. 2009;10(10):704-14.

5. Costa PM, Pedroso de Lima MC. MicroRNAs as molecular targets for cancer therapy: on the modulation of microRNA expression. Pharmaceuticals (Basel). 2013;6(10):1195-220.

6. Kasinski AL, Slack FJ. Epigenetics and genetics. MicroRNAs en route to the clinic: progress in validating and targeting microRNAs for cancer therapy. Nat Rev Cancer. 2011;11(12):849-64.

7. Hollingsworth MA, Swanson BJ. Mucins in cancer: protection and control of the cell surface. Nat Rev Cancer. 2004;4(1):45-60.

8. Bafna S, Kaur S, Batra SK. Membrane-bound mucins: the mechanistic basis for alterations in the growth and survival of cancer cells. Oncogene. 2010;29(20):2893-904.

9. Singh PK, Hollingsworth MA. Cell surface-associated mucins in signal transduction. Trends Cell Biol. 2006;16(9):467-76.

10.• Kaur S, Kumar S, Momi N, Sasson AR, Batra SK. Mucins in pancreatic cancer and its microenvironment. Nat Rev Gastroenterol Hepatol. 2013;10(10):607-20. This review discusses the pancreas specific expression pattern and role of various mucins under healthy, inflammatory, and cancerous conditions; diagnostic and/or prognostic efficacy of mucins; and mucin-based therapeutic strategies. The contribution of the tumour microenvironment in pancreatic cancer development and/or progression is also mentioned.

11. Kufe DW. Mucins in cancer: function, prognosis and therapy. Nat Rev Cancer. 2009;9(12):874-85.

12. Torres MP, Chakraborty S, Souchek J, Batra SK. Mucin-based targeted pancreatic cancer therapy. Curr Pharm Des. 2012;18(17): 2472-81.

13. Sachdeva M, Mo YY. MicroRNA-145 suppresses cell invasion and metastasis by directly targeting mucin 1 . Cancer Res. 2010;70(1): 378-87.

14. Wu H, Xiao Z, Wang K, Liu W, Hao Q. MiR-145 is downregulated in human ovarian cancer and modulates cell growth and invasion by targeting p70S6K1 and MUC1. Biochem Biophys Res Commun. 2013;441(4):693-700.

15. Jin C, Rajabi H, Kufe D. miR-1226 targets expression of the mucin 1 oncoprotein and induces cell death. Int J Oncol. 2010;37(1):61-9.

16. Rajabi H, Jin C, Ahmad R, McClary C, Joshi MD, Kufe D. Mucin 1 oncoprotein expression is suppressed by the miR-125b oncomir. Genes Cancer. 2010;1(1):62-8.

17. Rajabi H, Joshi MD, Jin C, Ahmad R, Kufe D. Androgen receptor regulates expression of the MUC1-C oncoprotein in human prostate cancer cells. Prostate. 2011;71(12):1299-308.

18. Martinez-Anton A, Sokolowska M, Kern S, Davis AS, Alsaaty S, Taubenberger JK, et al. Changes in microRNA and mRNA expression with differentiation of human bronchial epithelial cells. Am J Respir Cell Mol Biol. 2013;49(3):384-95.

19. Rotkrua P, Akiyama Y, Hashimoto Y, Otsubo T, Yuasa Y. MiR-9 downregulates CDX2 expression in gastric cancer cells. Int $\mathrm{J}$ Cancer. 2011;129(11):2611-20. 
20. Kozinn SI, Harty NJ, Delong JM, Deliyiannis C, Logvinenko T, Summerhayes IC, et al. MicroRNA profile to predict gemcitabine resistance in bladder carcinoma cell lines. Genes Cancer. 2013;4(12):61-9.

21. Srivastava SK, Bhardwaj A, Singh S, Arora S, Wang B, Grizzle WE, et al. MicroRNA-150 directly targets MUC4 and suppresses growth and malignant behavior of pancreatic cancer cells. Carcinogenesis. 2011;32(12):1832-9.

22.• Arora S, Swaminathan SK, Kirtane A, Srivastava SK, Bhardwaj A, Singh S, et al. Synthesis, characterization, and evaluation of poly (D,L-lactide-co-glycolide)-based nanoformulation of miRNA-150: potential implications for pancreatic cancer therapy. Int $\mathbf{J}$ Nanomedicine. 2014;9:2933-42. The paper presents a new, safe and effective nanovector platform for miR-150 delivery in pancreatic tumor cells to downregulate MUC4 expression and its downstream signaling; suppressing their growth, clonogenicity, motility, and invasion and thus highlights an effective pancreatic cancer therapeutic approach.

23. Wang WH, Chen J, Zhao F, Zhang BR, Yu HS, Jin HY, et al. MiR150-5p suppresses colorectal cancer cell migration and invasion through targeting MUC4. Asian Pac J Cancer Prev. 2014;15(15): 6269-73.

24.• Radhakrishnan P, Mohr AM, Grandgenett PM, Steele MM, Batra SK, Hollingsworth MA. MicroRNA-200c modulates the expression of MUC4 and MUC16 by directly targeting their coding sequences in human pancreatic cancer. PLoS One. 2013;8(10):e73356. This paper highlights that miR200 c not only regulates EMT modulating proteins but also downregulates expression of two aberrantly overexpressed transmembrane mucins (MUC4 and MUC16) associated with pancreatic cancer. This suggests a future possibility to utilize a single miRNA to target multiple mucins in a malignancy.

25. Lahdaoui F, Delpu Y, Vincent A, Renaud F, Messager M, Duchene B et al. miR-219-1-3p is a negative regulator of the mucin MUC4 expression and is a tumor suppressor in pancreatic cancer. Oncogene. 2014;0:1-9

26. Zhong T, Perelman JM, Kolosov VP, Zhou XD. MiR-146a negatively regulates neutrophil elastase-induced MUC5AC secretion from 16HBE human bronchial epithelial cells. Mol Cell Biochem. 2011;358(1-2):249-55.

27. Skrzypek K, Tertil M, Golda S, Ciesla M, Weglarczyk K, Collet G, et al. Interplay between heme oxygenase-1 and miR-378 affects non-small cell lung carcinoma growth, vascularization, and metastasis. Antioxid Redox Signal. 2013;19(7):644-60.

28. Al-Nakhle H, Burns PA, Cummings M, Hanby AM, Hughes TA, Satheesha $\mathrm{S}$, et al. Estrogen receptor $\{$ beta $\} 1$ expression is regulated by miR-92 in breast cancer. Cancer Res. 2010;70(11):4778-84.

29. Peng DX, Luo M, Qiu LW, He YL, Wang XF. Prognostic implications of microRNA-100 and its functional roles in human epithelial ovarian cancer. Oncol Rep. 2012;27(4):1238-44.

30. Kitamoto S, Yamada N, Yokoyama S, Houjou I, Higashi M, Goto $\mathrm{M}$, et al. DNA methylation and histone H3-K9 modifications contribute to MUC17 expression. Glycobiology. 2011;21(2):247-56.

31. Rachagani S, Kumar S, Batra SK. MicroRNA in pancreatic cancer: pathological, diagnostic and therapeutic implications. Cancer Lett. 2010;292(1):8-16.

32. Calin GA, Dumitru CD, Shimizu M, Bichi R, Zupo S, Noch E, et al. Frequent deletions and down-regulation of micro-RNA genes miR15 and miR16 at 13q14 in chronic lymphocytic leukemia. Proc Natl Acad Sci U S A. 2002;99(24):15524-9.

33. Lu J, Getz G, Miska EA, Alvarez-Saavedra E, Lamb J, Peck D, et al. MicroRNA expression profiles classify human cancers. Nature. 2005;435(7043):834-8

34. Sotillo E, Thomas-Tikhonenko A. Shielding the messenger (RNA): microRNA-based anticancer therapies. Pharmacol Ther. 2011;131(1):18-32.
35. Ota A, Tagawa H, Karnan S, Tsuzuki S, Karpas A, Kira S, et al. Identification and characterization of a novel gene, $\mathrm{C} 13$ or $\mathrm{f} 25$, as a target for 13q31-q32 amplification in malignant lymphoma. Cancer Res. 2004;64(9):3087-95.

36. Volinia S, Calin GA, Liu CG, Ambs S, Cimmino A, Petrocca F, et al. A microRNA expression signature of human solid tumors defines cancer gene targets. Proc Natl Acad Sci U S A. 2006;103(7):2257-61.

37. Yan LX, Huang XF, Shao Q, Huang MY, Deng L, Wu QL, et al. MicroRNA miR-21 overexpression in human breast cancer is associated with advanced clinical stage, lymph node metastasis and patient poor prognosis. RNA. 2008;14(11):2348-60.

38. Hua Z, Lv Q, Ye W, Wong CK, Cai G, Gu D, et al. MiRNA-directed regulation of VEGF and other angiogenic factors under hypoxia. PLoS One. 2006;1:e116.

39. Kim VN. MicroRNA biogenesis: coordinated cropping and dicing. Nat Rev Mol Cell Biol. 2005;6(5):376-85.

40. Lee Y, Kim M, Han J, Yeom KH, Lee S, Baek SH, et al. MicroRNA genes are transcribed by RNA polymerase II. EMBO J. 2004;23(20):4051-60.

41. Han J, Lee Y, Yeom KH, Nam JW, Heo I, Rhee JK, et al. Molecular basis for the recognition of primary microRNAs by the DroshaDGCR8 complex. Cell. 2006;125(5):887-901.

42. Bohnsack MT, Czaplinski K, Gorlich D. Exportin 5 is a RanGTPdependent dsRNA-binding protein that mediates nuclear export of pre-miRNAs. RNA. 2004;10(2):185-91.

43. Chendrimada TP, Gregory RI, Kumaraswamy E, Norman J, Cooch N, Nishikura K, et al. TRBP recruits the Dicer complex to Ago2 for microRNA processing and gene silencing. Nature. 2005;436(7051):740-4.

44. Khvorova A, Reynolds A, Jayasena SD. Functional siRNAs and miRNAs exhibit strand bias. Cell. 2003;115(2):209-16.

45. Heneghan HM, Miller N, Kerin MJ. MiRNAs as biomarkers and therapeutic targets in cancer. Curr Opin Pharmacol. 2010;10(5): 543-50.

46. Nath S, Mukherjee P. MUC1: a multifaceted oncoprotein with a key role in cancer progression. Trends Mol Med. 2014;20(6):332-42.

47. Gotte M, Mohr C, Koo CY, Stock C, Vaske AK, Viola M, et al. miR-145-dependent targeting of junctional adhesion molecule A and modulation of fascin expression are associated with reduced breast cancer cell motility and invasiveness. Oncogene. 2010;29(50):6569-80.

48. Zhang J, Guo H, Zhang H, Wang H, Qian G, Fan X, et al. Putative tumor suppressor miR-145 inhibits colon cancer cell growth by targeting oncogene Friend leukemia virus integration 1 gene. Cancer. 2011;117(1):86-95.

49. Bloomston M, Frankel WL, Petrocca F, Volinia S, Alder H, Hagan JP, et al. MicroRNA expression patterns to differentiate pancreatic adenocarcinoma from normal pancreas and chronic pancreatitis. JAMA. 2007;297(17):1901-8.

50. Bousquet M, Quelen C, Rosati R, Mansat-De M, La SR V, Bastard C. Myeloid cell differentiation arrest by miR-125b-1 in myelodysplastic syndrome and acute myeloid leukemia with the $\mathrm{t}(2 ; 11)(\mathrm{p} 21 ; \mathrm{q} 23)$ translocation. J Exp Med. 2008;205(11):2499-506.

51. Iorio MV, Ferracin M, Liu CG, Veronese A, Spizzo R, Sabbioni S, et al. MicroRNA gene expression deregulation in human breast cancer. Cancer Res. 2005;65(16):7065-70.

52. Xu X, Wells A, Padilla MT, Kato K, Kim KC, Lin Y. A signaling pathway consisting of miR-551b, catalase and MUC1 contributes to acquired apoptosis resistance and chemoresistance. Carcinogenesis. 2014;35(11):2457-66.

53. Ponnusamy MP, Seshacharyulu P, Lakshmanan I, Vaz AP, Chugh S, Batra SK. Emerging role of mucins in epithelial to mesenchymal transition. Curr Cancer Drug Targets. 2013;13(9):945-56.

54. Bafna S, Kaur S, Momi N, Batra SK. Pancreatic cancer cells resistance to gemcitabine: the role of MUC4 mucin. Br J Cancer. 2009;101(7):1155-61. 
55. Ma Y, Zhang P, Wang F, Zhang H, Yang J, Peng J, et al. miR-150 as a potential biomarker associated with prognosis and therapeutic outcome in colorectal cancer. Gut. 2012;61(10):1447-53.

56. Haridas D, Ponnusamy MP, Chugh S, Lakshmanan I, Seshacharyulu P, Batra SK. MUC16: molecular analysis and its functional implications in benign and malignant conditions. FASEB J. 2014;28(10):4183-99.

57. Comamala M, Pinard M, Theriault C, Matte I, Albert A, Boivin M, et al. Downregulation of cell surface CA125/MUC16 induces epithelial-to-mesenchymal transition and restores EGFR signalling in NIH:OVCAR3 ovarian carcinoma cells. Br J Cancer. 2011;104(6):989-99.

58. Lakshmanan I, Ponnusamy MP, Das S, Chakraborty S, Haridas D, Mukhopadhyay P, et al. MUC16 induced rapid G2/M transition via interactions with JAK2 for increased proliferation and antiapoptosis in breast cancer cells. Oncogene. 2012;31(7):805-17.

59. Senapati S, Ho SB, Sharma P, Das S, Chakraborty S, Kaur S, et al. Expression of intestinal MUC17 membrane-bound mucin in inflammatory and neoplastic diseases of the colon. J Clin Pathol. 2010;63(8):702-7.

60. Moniaux N, Junker WM, Singh AP, Jones AM, Batra SK. Characterization of human mucin MUC17. Complete coding sequence and organization. J Biol Chem. 2006;281(33):23676-85.

61. Hirono S, Yamaue H, Hoshikawa Y, Ina S, Tani M, Kawai M, et al. Molecular markers associated with lymph node metastasis in pancreatic ductal adenocarcinoma by genome-wide expression profiling. Cancer Sci. 2010;101(1):259-66.

62. Evans CM, Koo JS. Airway mucus: the good, the bad, the sticky. Pharmacol Ther. 2009;121(3):332-48.

63. Bu X, Li L, Li N, Tian X, Huang P. Suppression of mucin 2 enhances the proliferation and invasion of LS174T human colorectal cancer cells. Cell Biol Int. 2011;35(11):1121-9.

64. Velcich A, Yang W, Heyer J, Fragale A, Nicholas C, Viani S, et al. Colorectal cancer in mice genetically deficient in the mucin Muc2. Science. 2002;295(5560):1726-9.

65. Boltin D, Niv Y. Mucins in gastric cancer-an update. J Gastrointest Dig Syst. 2013;3(123):15519.

66. Turner J, Jones CE. Regulation of mucin expression in respiratory diseases. Biochem Soc Trans. 2009;37(Pt 4):877-81.

67. Yu CJ, Yang PC, Shun CT, Lee YC, Kuo SH, Luh KT. Overexpression of MUC5 genes is associated with early postoperative metastasis in non-small-cell lung cancer. Int $\mathrm{J}$ Cancer. 1996;69(6):457-65.

68. Aishima S, Kuroda Y, Nishihara Y, Taguchi K, Taketomi A, Maehara Y, et al. Gastric mucin phenotype defines tumour progression and prognosis of intrahepatic cholangiocarcinoma: gastric foveolar type is associated with aggressive tumour behaviour. Histopathology. 2006;49(1):35-44.
69. Takikita M, Altekruse S, Lynch CF, Goodman MT, Hernandez BY, Green M, et al. Associations between selected biomarkers and prognosis in a population-based pancreatic cancer tissue microarray. Cancer Res. 2009;69(7):2950-5.

70. Yan F, Li W, Zhou H, Wu Y, Ying S, Chen Z, et al. Interleukin-13induced MUC5AC expression is regulated by a PI3K-NFAT3 pathway in mouse tracheal epithelial cells. Biochem Biophys Res Commun. 2014;446(1):49-53.

71. Kumar M, Ahmad T, Sharma A, Mabalirajan U, Kulshreshtha A, Agrawal A, et al. Let-7 microRNA-mediated regulation of IL-13 and allergic airway inflammation. J Allergy Clin Immunol. 2011;128(5):1077-85.

72. Lee YS, Kim HK, Chung S, Kim KS, Dutta A. Depletion of human micro-RNA miR-125b reveals that it is critical for the proliferation of differentiated cells but not for the down-regulation of putative targets during differentiation. J Biol Chem. 2005;280(17):16635-41.

73. Kloosterman WP, Lagendijk AK, Ketting RF, Moulton JD, Plasterk RH. Targeted inhibition of miRNA maturation with morpholinos reveals a role for miR-375 in pancreatic islet development. PLoS Biol. 2007;5(8):e203.

74. Thorsen SB, Obad S, Jensen NF, Stenvang J, Kauppinen S. The therapeutic potential of microRNAs in cancer. Cancer J. 2012;18(3):275-84.

75. Kluiver J, Gibcus JH, Hettinga C, Adema A, Richter MK, Halsema $\mathrm{N}$, et al. Rapid generation of microRNA sponges for microRNA inhibition. PLoS One. 2012;7(1):e29275.

76. Li L, Xie X, Luo J, Liu M, Xi S, Guo J, et al. Targeted expression of miR-34a using the T-VISA system suppresses breast cancer cell growth and invasion. Mol Ther. 2012;20(12):2326-34.

77. Trang P, Wiggins JF, Daige CL, Cho C, Omotola M, Brown D, et al. Systemic delivery of tumor suppressor microRNA mimics using a neutral lipid emulsion inhibits lung tumors in mice. Mol Ther. 2011;19(6):1116-22.

78. Costa PM, Cardoso AL, Mendonca LS, Serani A, Custodia C, Conceicao M, et al. Tumor-targeted chlorotoxin-coupled nanoparticles for nucleic acid delivery to glioblastoma cells: a promising system for glioblastoma treatment. Mol Ther Nucleic Acids. 2013;2:e100.

79. Dai F, Zhang Y, Zhu X, Shan N, Chen Y. Anticancer role of MUC1 aptamer-miR-29b chimera in epithelial ovarian carcinoma cells through regulation of PTEN methylation. Target Oncol. 2012;7(4):217-25.

80. Dai F, Zhang Y, Zhu X, Shan N, Chen Y. The anti-chemoresistant effect and mechanism of MUC1 aptamer-miR-29b chimera in ovarian cancer. Gynecol Oncol. 2013;131(2):451-9.

81. Liu N, Zhou C, Zhao J, Chen Y. Reversal of paclitaxel resistance in epithelial ovarian carcinoma cells by a MUC1 aptamer-let-7i chimera. Cancer Invest. 2012;30(8):577-82. 